\title{
Measurements of the indium hyperfine structure in an atmospheric-pressure flame by use of diode-laser-induced fluorescence
}

\author{
Johan Hult, Iain Stewart Burns, and Clemens Friedrich Kaminski \\ Department of Chemical Engineering, University of Cambridge, Pembroke Street, Cambridge CB2 3RA, UK
}

Received September 19, 2003

\begin{abstract}
We report on what we believe is the first demonstration of laser-induced fluorescence (LIF) in flames by use of diode lasers. Indium atoms seeded into an atmospheric-pressure flame at trace concentrations are excited by a blue GaN laser operating near $410 \mathrm{~nm}$. The laser is mounted in an external-cavity configuration, and the hyperfine spectrum of the $5^{2} P_{1 / 2} \rightarrow 6^{2} S_{1 / 2}$ transition is captured at high resolution in single-wavelength sweeps lasting less than one tenth of a second. The research demonstrates the potential of diode-based LIF for practical diagnostics of high-temperature reactive flows. (C) 2004 Optical Society of America

OCIS codes: $300.2530,120.1740,300.6260,020.2930$.
\end{abstract}

Laser-induced fluorescence (LIF) is one of the most useful tools for the diagnostics of combusting flows. ${ }^{1}$ It is highly species selective and sensitive and capable of measuring concentrations and temperature with excellent spatial and temporal resolution. LIF has an important role in the investigation of fundamental combustion phenomena, the validation of numerical models, and the development of more-efficient combustion devices. ${ }^{2}$ In flames, LIF has been conducted almost exclusively with high-power pulsed-laser devices to improve signal levels in the presence of high background luminosities and strong signal quenching caused by molecular collisions. However, continuous LIF measurement techniques have also been applied in flames in the past, employing continuouswave dye lasers. ${ }^{3,4}$ The large and expensive equipment required prevents routine application of LIF in industrial combustion environments or for process control applications. Diode lasers, on the other hand, represent an attractive alternative for the development of practical flame sensors. They are rapidly tunable, compact, reliable, robust, and cheap. In the near-infrared spectral region, diode lasers are already being extensively used for concentration and temperature measurements based on line-of-sight absorption techniques. ${ }^{5-8}$ Only recently, however, have devices become available in the blue spectral region ${ }^{9}$ that have the potential to excite electronic transitions in flame-generated and flame-seeded species directly. In this Letter we report on the first application of a $\mathrm{GaN}$ diode laser operating near $410 \mathrm{~nm}$ to probe indium atoms seeded into flames by LIF. A single-mode external-cavity laser was built, and spectra resolving the hyperfine structure of the $5^{2} P_{1 / 2} \rightarrow 6^{2} S_{1 / 2}$ transition were recorded in single-wavelength sweeps at $20-\mathrm{Hz}$ repetition rates. To our knowledge, this represents the first speciesspecific LIF measurement in a flame that is based on diode laser technology. Theoretical spectra were fitted to the high-resolution data obtained, from which accurate line-shape information could be deduced. The potential of employing diode lasers for flame tem- perature diagnostics, utilizing established continuous LIF measurement techniques, ${ }^{3,4}$ is discussed.

A 410-nm Fabry-Perot (FP) diode laser (Nichia Corporation) was configured in a specially designed external cavity. An 1800-lines/mm holographic grating was mounted in a Littrow configuration to serve as a frequency-selective device and output coupler of the external laser cavity. ${ }^{10,11}$ The external-cavity tuning scheme employed ${ }^{12}$ resulted in a mode-hop-free tuning range exceeding $50 \mathrm{GHz}$, without the need for antireflection coating of the FP diode crystal facets. At the wavelengths used for the LIF experiments the output power of the external-cavity diode laser was approximately $2 \mathrm{~mW}$.

Experiments were conducted in a 50-mm-diameter atmospheric-pressure methanol pool flame. The methanol was seeded with $\mathrm{InCl}_{3}\left(80\right.$ parts in $10^{6}$ ) to generate indium atoms in the flame. The laser beam was focused by a 300-mm lens into the flame, and fluorescence signals were collected at right angles and imaged $f_{\#}=1$ onto a $0.65-\mathrm{mm}$ pinhole in front of a filtered photomultiplier tube (Hamamatsu, R3788). The imaged interaction volume corresponded to a cylinder of approximately $85 \mu \mathrm{m}$ in diameter and $0.65 \mathrm{~mm}$ in length. A fraction of the laser light was picked off by a glass plate and passed through a solid FP etalon (free spectral range of $3.1 \mathrm{GHz}$ ) to calibrate the wavelength of the laser scan. A reflection from a second glass plate was used to monitor the output power of the laser. Mode hops occurring at the ends of the scanning range were observable as distinct disruptions on the otherwise continuous etalon and laser power traces. The LIF and reference signals were acquired by a 16-bit PC data acquisition card (National Instruments, PCI-6014).

Indium was excited in the $5^{2} P_{1 / 2} \rightarrow 6^{2} S_{1 / 2}$ transition system near $410 \mathrm{~nm}$, and the filtered fluorescence signal corresponding to the $6^{2} S_{1 / 2} \rightarrow 5^{2} P_{3 / 2}$ transition near $451 \mathrm{~nm}$ was recorded. An interference bandpass filter (CVI, FWHM $=10 \mathrm{~nm}$, center $=450 \mathrm{~nm}$ ) was used to reject flame emission and scatter from the excitation laser. The laser wavelength was scanned at 
$20 \mathrm{~Hz}$ over a $50-\mathrm{GHz}$ tuning range to cover the four hyperfine transitions in the $5^{2} P_{1 / 2} \rightarrow 6^{2} S_{1 / 2}$ system.

Because the transition strengths of atomic species are very strong, even modest laser power will result in a strong fluorescence signal. In the flame the indium fluorescence is clearly visible by eye, and in Fig. 1 a photo of the fluorescence signal in the flame is shown (40-ms exposure time).

In Fig. 2 a 48-GHz-wide single-excitation scan recorded in $50 \mathrm{~ms}$ is shown. The indium atoms are excited from the $5^{2} P_{1 / 2}$ ground level to the $6^{2} S_{1 / 2}$ excited state, and they can then decay back into either the $5^{2} P_{1 / 2}$ state by emitting 410 -nm light or into the $5^{2} P_{3 / 2}$ state by emitting $451-\mathrm{nm}$ fluorescence light (see inset in Fig. 2). The peaks in the spectrum correspond to transitions between the hyperfine levels in the $5^{2} P_{1 / 2}$ and $6^{2} S_{1 / 2}$ states. The hyperfine splitting in the ground state is $11.4 \mathrm{GHz}$, and in the excited state it is $8.4 \mathrm{GHz} .{ }^{13}$ The left peak corresponds to the $F=5 \rightarrow F^{\prime}=4$ transition and the right peak to the $4 \rightarrow 5^{\prime}$ transition. The center peak consists of two transitions $\left(5 \rightarrow 5^{\prime}\right.$ and $\left.4 \rightarrow 4^{\prime}\right)$, which appear merged because they are separated by only $3 \mathrm{GHz}$, which is less than the widths of their flame-broadened line shapes.

A spectrum averaged over 50 scans of the laser frequency is also shown in Fig. 2. The individual spectra were normalized by the simultaneously recorded laser output power before averaging. Power changes slowly by approximately $15 \%$ during the scan over the shown wavelength range. The shape of the spectrum indicates that pressure broadening is the dominant broadening mechanism in the present flame. This is confirmed by Voigt profiles fitted to the averaged spectra, because the resulting Lorentzian contribution is approximately four times larger than the Doppler width. A nonlinear least-squares fit of four Voigt profiles is shown as a dashed curve in Fig. 2, and the four individual profiles are also shown. The measured separations of the last three peaks with respect to the first one are $8.5,11.7$, and $19.8 \mathrm{GHz}$, which closely match the expected separations: 8.4, 11.4, and $19.8 \mathrm{GHz}$. The relative peak heights of the four fitted profiles are 1:0.69:0.33:0.92, from left to right, which agrees fairly well with the relative ratios calculated from the Clebsch-Gordan coefficients. ${ }^{14}$ These coefficients are a function of the $F, J$, and $I$ quantum numbers of the hyperfine states involved in the transition; for the $5^{2} P_{1 / 2} \rightarrow 6^{2} S_{1 / 2}$ indium transition the evaluated coefficients lead to theoretical relative ratios of 1:0.67:0.36:1. The excellent agreement demonstrates the fidelity of the technique.

The pressure-broadening contribution to the linewidth of the Voigt profiles shown in Fig. 2 is approximately $7.3 \mathrm{GHz}$. As a comparison, the expected pressure broadening of indium in a pure $\mathrm{N}_{2}$ gas, which is the most abundant species in the flame, at a temperature of $1500 \mathrm{~K}$ is $6.3 \mathrm{GHz}$. This estimate is based on indium line-broadening rates reported by Eberz et al..$^{15}$ and uses the impact pressure-broadening approximation. ${ }^{16}$ The larger observed pressure broadening compared with the estimated broadening could be due to three different factors: A temperature $T$ at the measurement location that is lower than $1500 \mathrm{~K}$ would lead to a correspondingly larger pressure broadening $\Delta \nu$ because $\Delta \nu \sim T^{-\eta}$ ( $\eta$ is the temperature exponent for collision broadening), slightly higher broadening coefficients for species other than $\mathrm{N}_{2}$ compared with the coefficient for $\mathrm{N}_{2}$ would also lead to a larger observed broadening, and a value of $\eta$ lower than the 0.7 predicted by the impact approximation would lead to a larger value of the estimated broadening. ${ }^{15}$

The strong signals obtained demonstrate a clear potential for fluorescence-based temperature measurements by use of indium-based two-line atomic fluorescence (TLAF).,17 This would, for the first time to our knowledge, allow the use of diode lasers for spatially precise temperature measurements, avoiding averaging effects normally associated with absorption-based temperature techniques. It would extend the use of diode lasers as temperature sensors to nonhomogeneous flow conditions present in most practical combustion devices. In TLAF the population distribution of two temperature-sensitive electronic states of atoms seeded into a flame is probed by excitation to a common upper state. Temperature is then evaluated from the ratio of the corresponding fluorescence signals from this excited state. ${ }^{4,18}$ It has been shown that indium is an excellent seed species

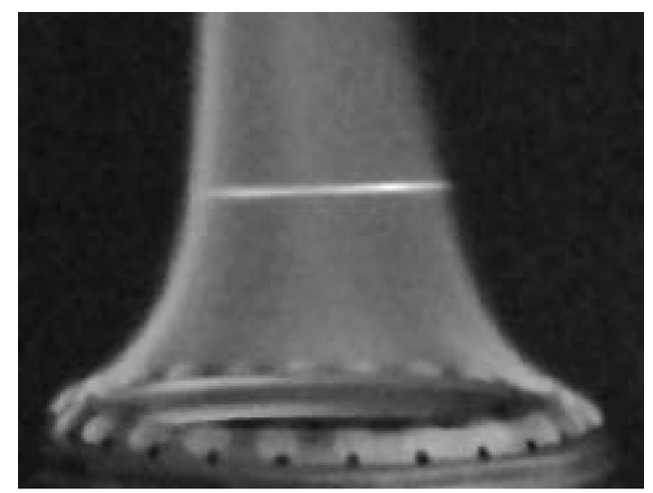

Fig. 1. Photo of flame and indium LIF signal along the laser beam.

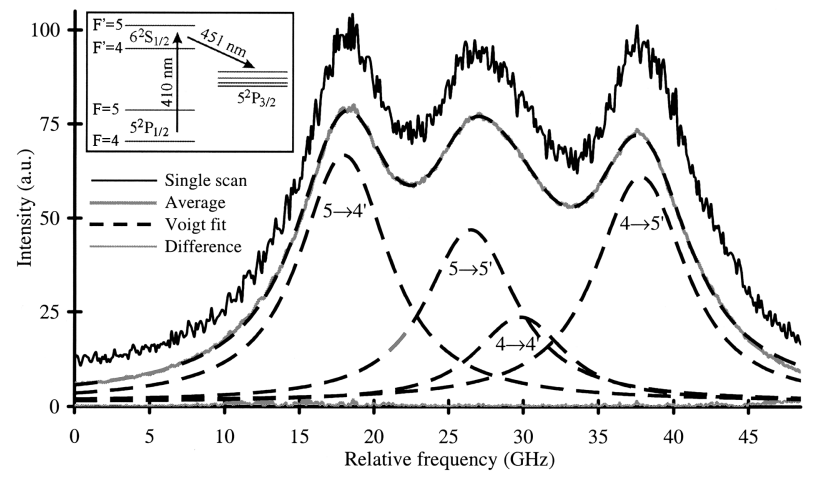

Fig. 2. LIF spectrum of the $5^{2} P_{1 / 2} \rightarrow 6^{2} S_{1 / 2}$ indium hyperfine structure obtained from an indium-seeded flame. A single-scan spectrum is shown, as well as an average over 50 scans of the diode laser output wavelength. Dashed curves, fit of four Voigt profiles to the average spectrum, corresponding to the four $5^{2} P_{1 / 2} \rightarrow 6^{2} S_{1 / 2}$ hyperfine transitions. Inset, indium energy level scheme. 
for TLAF, because the spin-orbit splitting in the $5 P$ lower states matches the temperature range covering most practical combustion conditions, and this leads to excellent temperature sensitivity of their relative populations. ${ }^{19}$

Indium TLAF temperature measurements have been reported in the past by use of either pulsed Nd:YAG or continuous $\mathrm{Ar}^{+}$laser-pumped dye laser systems. ${ }^{4,17} \mathrm{~A}$ diode-laser-based sensor would provide a compact and cost-efficient alternative to the laser systems previously used. Furthermore, more accurate temperature measurements are feasible, because the single-mode laser source would eliminate the noise caused by laser mode fluctuations that affect multimode systems. ${ }^{4}$

In Fig. 2 it is seen that at atmospheric pressure and elevated temperatures the four hyperfine peaks combine to form an almost 30-GHz-wide, quasihomogeneously broadened transition from the ground state to the excited state. This is much wider than the width estimated in previous TLAF work on indium. In previous work the assumption has been made that the laser linewidth is much larger than the atomic linewidth in the studied flames, ${ }^{4,17}$ which, as the present research shows, is clearly not valid. This is particularly the case for measurements reported in engines, where pressures up to $25 \mathrm{~atm}$ are encountered, leading to severe line-broadening effects. The observed linewidth can be an advantage for TLAF applications, however, because the effect of pressure-dependent line shifts on spectral overlapping between laser and transition profiles will be reduced. The effect on the accuracy of previous temperature measurements based on the technique is currently being investigated but is believed to be small. For constant pressure conditions the linewidths extracted from curve fits, such as the one in Fig. 2, could be used to deduce flame temperatures directly ${ }^{20}$; however, this requires a calibration measurement of the pressure broadening at a well-defined temperature.

Our results show that blue diode lasers can be used as efficient high-resolution excitation sources for LIF from atoms seeded to flames at trace levels. By use of an external-cavity diode laser operating around $410 \mathrm{~nm}$, the hyperfine structure of indium was successfully resolved. The line broadening for typical flame conditions could be estimated and was found to be strongly dominated by pressure broadening. Diode lasers operating near $451 \mathrm{~nm}$ are also becoming available. This means that the $5^{2} P_{3 / 2} \rightarrow 6^{2} S_{1 / 2}$ transition can also be targeted, and research using such a laser diode is currently being undertaken in the authors' laboratory. If successful, TLAF flame thermometry based on diode sensors will become feasible in the future.

This work was supported by grants from the Paul Instrument Fund of the Royal Society. J. Hult was supported by a Marie Curie Fellowship of the European Community Human Potential program under contract HPMF-CT-2002-01574. I. S. Burns was supported by an Engineering and Physical Sciences Research Council Cooperative Award in Science and Engineering and partially funded by Rolls-Royce. Support from the Engineering and Physical Sciences Research Council under grants GR/R98679/01 and GR/R61994/ 01 is gratefully acknowledged. We thank P. Ewart for assistance and the loan of experimental equipment. A patent application has been filed for the new external-cavity diode laser technology. J. Hult's e-mail address is jfh36@cheng.cam.ac.uk.

\section{References}

1. J. W. Daily, Prog. Energy Combust. Sci. 23, 133 (1997).

2. J. Wolfrum, in Twenty-Seventh Symposium (International) on Combustion (The Combustion Institute, Pittsburgh, Pa., 1998), p. 1.

3. R. G. Joklik and J. W. Daily, Appl. Opt. 21, 4158 (1982).

4. J. E. Dec and J. O. Keller, in Twenty-First Symposium (International) on Combustion (The Combustion Institute, Pittsburgh, Pa., 1986), p. 1737.

5. R. M. Mihalcea, D. S. Baer, and R. K. Hanson, Meas. Sci. Technol. 9, 327 (1998).

6. M. G. Allen, Meas. Sci. Technol. 9, 545 (1998).

7. S. T. Sanders, J. A. Baldwin, T. P. Jenkins, D. S. Baer, and R. K. Hanson, in Proceedings of the Combustion Institute, Vol. 28 (The Combustion Institute, Pittsburgh, Pa., 2000), p. 587.

8. V. Ebert, T. Fernhoz, C. Giesemann, H. Pitz, H. Teichert, J. Wolfrum, and H. Jaritz, in Proceedings of the Combustion Institute, Vol. 28 (The Combustion Institute, Pittsburgh, Pa., 2000), p. 423.

9. S. Nakamura, Science 281, 956 (1998).

10. K. B. MacAdam, A. Steinbach, and C. Wieman, Am. J. Phys. 60, 1098 (1992).

11. U. Gustafsson, J. Alnis, and S. Svanberg, Am. J. Phys. 68, 660 (2000).

12. J. Hult, I. S. Burns, and C. F. Kaminski are preparing a manuscript to be called "A wide continuous tuning range blue external cavity diode laser system."

13. H. Leinen, D. Gläßner, H. Metcalf, R. Wynands, D. Haubrich, and D. Meschede, Appl. Phys. B 70, 567 (2000).

14. C. Candler, Atomic Spectra and the Vector Model (Hilger, London, 1964).

15. J. Eberz, G. Huber, T. Kühl, and G. Ulm, J. Phys. B 17, 3075 (1984).

16. I. I. Sobelman, L. A. Vainshtein, and E. A. Yukov, Excitation of Atoms and Broadening of Spectral Lines (Springer-Verlag, Berlin, 1981).

17. C. F. Kaminski, J. Engström, and M. Aldén, in TwentySeventh Symposium (International) on Combustion (The Combustion Institute, Pittsburgh, Pa., 1998), p. 85.

18. A. C. Eckbreth, Laser Diagnostics for Combustion Temperature and Species, 2nd ed. (Gordon \& Breach, Amsterdam, 1996).

19. J. Engström, C. F. Kaminski, M. Aldén, G. Josefsson, and I. Magnusson, in 1999 SAE International Congress and Exposition (Society of Automobile Engineers, Warrendale, Pa., 1999), paper 1999-01-3541.

20. S. T. Sanders, D. W. Mattison, L. Ma, J. B. Jeffries, and R. K. Hanson, Opt. Express 10, 505 (2002), http://www.opticsexpress.org. 